\title{
Violência obstétrica: fatos relatados na prática e os impactos na vida da mulher
}

\author{
Obstetric violence: facts reported in practice and impacts on women's lives
}

Violencia obstétrica: hechos denunciados en la práctica e impactos en la vida de las mujeres

Bianca Fachetti Carvalho ${ }^{1 *}$, Raiane Antunes Sampaio ${ }^{1}$, Regiany Rodrigues Silva ${ }^{1}$, João Pedro Pereira de Carvalho', Jordana Vaz de Abreu${ }^{1}$, Rhafaela Souza Oliveira ${ }^{1}$, Lucas Antonio Otoni Batista ${ }^{1}$, Julia Vallentina de Souza Saturnino ${ }^{1}$, Caroline Martins Soares ${ }^{1}$, Evelyn Corrêa de Oliveira ${ }^{1}$.

\section{RESUMO}

Objetivo: Realizar uma revisão acerca do impacto do parto clínico e medicalizado na vida da mulher, bem como a importância de informações adequadas durante o pré-parto para evitar a violência obstétrica. Revisão bibliográfica: Até o final do século XVIII, os partos eram realizados por parteiras, porém, com a implantação das escolas médicas, houve uma mudança desse cenário, tornando altamente medicalizado e com procedimentos rotineiros, o que deu origem a violência obstétrica. Uma série de violações dos direitos e perda da autonomia da mulher, fez com que a Organização Mundial da Saúde (OMS) e o Sistema Único de Saúde (SUS) criassem políticas e programas de saúde voltada a práticas humanizadas, assim como leis que garantem o desejo da parturiente, visando diminuir os procedimentos. Cesáreas, episiotomia, manobra de Kristeller e uso de ocitocina são alguns deles, que precisam ser discutidos durante o pré, intra e pósparto, pois criam medo e insegurança na família, devido ao histórico marcado por uma violência e desumanização. Considerações finais: Portanto, a participação ativa dos profissionais da saúde aliado a uma assistência humanizada, são fundamentais para diminuir os números alarmantes de violência obstétrica, e consequentemente, diminuir as sequelas que são causadas na vida reprodutiva e sexual da mulher.

Palavras-chave: Parto humanizado, Assistência integral a saúde da mulher, Violência contra a mulher.

\begin{abstract}
Objective: To carry out a review of the impact of clinical and medicalized childbirth on women's lives, as well as the importance of adequate information during prepartum to avoid obstetric violence. Bibliographic review: Until the end of the 18th century, births were performed by midwives, however, with the implementation of medical schools, there was a change in this scenario, becoming highly medicalized and with routine procedures, which gave rise to obstetric violence. A series of violations of women's rights and loss of autonomy led the World Health Organization (WHO) and the Unified Health System (SUS) to create health policies and programs aimed at humanized practices, as well as laws that guarantee the desire of the parturient, aiming to reduce the procedures. Cesarean sections, episiotomy, Kristeller's maneuver and the use of oxytocin are some of them, which need to be discussed during pre, intra and postpartum, as they create fear and insecurity in the family, due to the history marked by violence and dehumanization. Final considerations: Therefore, the active participation of health professionals combined with humanized care are essential to reduce the alarming numbers of obstetric violence, and consequently, reduce the sequelae that are caused in the reproductive and sexual life of women.
\end{abstract}

Keywords: Humanized childbirth, Comprehensive women's health care, Violence against women.

${ }^{1}$ Universidade de Rio Verde (UniRV), Goianésia - GO. *E-mail: biancafachetti12@gmail.com

SUBMETIDO EM: 8/2021

ACEITO EM: 8/2021

PUBLICADO EM: 8/2021 


\section{RESUMEN}

Objetivo: Realizar una revisión del impacto del parto clínico y medicalizado en la vida de las mujeres, así como la importancia de contar con información adecuada durante el preparto para evitar la violencia obstétrica. Revisión bibliográfica: Hasta fines del siglo XVIII, los partos eran realizados por parteras, sin embargo, con la implementación de las facultades de medicina, se produjo un cambio en este escenario, volviéndose altamente medicalizados y con procedimientos rutinarios, que dieron lugar a la violencia obstétrica. Una serie de violaciones a los derechos de las mujeres y pérdida de autonomía llevó a la Organización Mundial de la Salud (OMS) y al Sistema Único de Salud (SUS) a crear políticas y programas de salud orientados a prácticas humanizadas, así como leyes que garanticen el deseo de la parturienta, con el objetivo de reducir los procedimientos. Las cesáreas, la episiotomía, la maniobra de Kristeller y el uso de oxitocina son algunos de ellos, que deben ser discutidos durante el pre, intra y posparto, ya que generan miedo e inseguridad en la familia, debido a la historia marcada por la violencia y la deshumanización. Consideraciones finales: Por lo tanto, la participación activa de los profesionales de la salud combinada con la atención humanizada es fundamentales para reducir las cifras alarmantes de violencia obstétrica y, en consecuencia, reducir las secuelas que se provocan en la vida reproductiva y sexual de las mujeres.

Palabras clave: Parto humanizado, Atención integral a la saude de la mujer, Violencia contra la mujer.

\section{INTRODUÇÃO}

As gestantes trazem consigo uma história pessoal, familiar e cultural que terá uma forte influência no trabalho de parto e no nascimento. Um marco entre ser filha (o) e tornar-se mãe, entre um bebê idealizado e um bebê realista, entre tantas outras expectativas e mudanças que ocorrem na vida da mulher durante esse período, abrangendo o campo físico, mental, emocional e social (PALHARINI LA e FIGUEIRÔA SDM, 2018).

Anteriormente, apesar dos poucos recursos oferecidos no parto, as mulheres possuíam maior autonomia sobre o seu corpo e isso tornava-o mais humanizado e natural. Os partos eram caseiros, com a assistência das mulheres da casa e da parteira, muitas vezes, recorrendo a chás e massagens para alívio da dor (BRASIL, 2010). Dessa forma, no início do século XIX com a implantação das escolas médicas no Brasil e o processo de mudanças na obstetrícia, respectivamente, o parto estava deixando de ser um "ritual" feminino para se tornar um evento médico (PIMENTA DG, et al., 2013).

Desde o século $X X$, começou a ter um aumento no número de partos realizados em hospitais e concomitante a ele, o surgimento de tecnologias, técnicas e condutas médicas inseridas durante o trabalho de parto, levando a acreditar que o parto se tornaria "mais seguro" e garantia um maior benefício para a saúde da mãe e do bebê (PALHARINI LA e FIGUEIRÔA SDM, 2018). Assim, com o propósito de ter uma assistência especializada e altamente tecnológica, fez com que o parto orgânico, tornasse um parto totalmente medicalizado, e não mais como uma exceção (VENDRUSCOLO CT, 2018; PALHARINI LA e FIGUEIRÔA SDM, 2018).

Então, com a mudança de um parto domiciliar para um avanço excessivo dos procedimentos hospitalares e estímulo por parte dos profissionais, percebe-se que as mulheres têm se tornado vítimas de violência, seja física, emocional ou sexual, configurando o que hoje é definido como violência obstétrica. Apesar de ser um termo novo, essas práticas vêm sendo realizadas desde a implantação do parto hospitalar no início do século XX (TESSER CD, et al., 2015).

De acordo com a Organização Mundial da Saúde (OMS), mulheres solteiras, adolescentes de baixo poder aquisitivo, migrantes e de minoria étnicas são mais propensas a sofrerem violência obstétrica (BRASIL, 2002). Como é o caso, que acontece com muitas pacientes de raça negra, que já possuem um histórico violentado, um pré-natal fragilizado e que tem como crença social de que são mais fortes e resistentes à dor, quando comparada à mulher branca (VENTURE G e GODINHO T, 2013).

Por meio da pesquisa "Mulheres brasileiras e gênero nos espaços públicos e privados" realizada em 2010, constatou-se que das 100 pessoas entrevistadas, 25 sofreram algum tipo de violência no atendimento 
ao parto, seja ela visível ou não. A violência psicológica, que é a forma mais comum nesse meio, é representada por gritos dentro da sala de parto, comandos e nomes que remetem inferioridade, negar atendimento ou informações e a imposição de dificuldades para que a gestante receba tal serviço. Além disso, existe a violência física que está relacionado ao ato de utilizar ocitocina, episiotomia, fórceps e outros, muitas vezes sem o consentimento da paciente (VENTURE G e GODINHO T, 2013).

Diante de toda a reformulação no cenário do nascimento, observa-se que a violência obstétrica traz consigo algumas complicações para o binômio mãe-bebê, desde medo a frustração por falta de humanização nesse momento tão único e delicado, que é o parto. Portanto, esse fato é considerado uma violação dos direitos humanos, uma vez que podem apresentar sequelas e traumas na vida da mulher, interferindo na saúde reprodutiva e sexual (ROCHA NFF e FERREIRA J, 2020; QUEIROZ FS, et al., 2020).

Dessa forma, a diminuição da incidência de violência obstétrica começa com uma mudança na assistência à parturiente, desde o pré-natal até o puerpério, oferecendo informações claras e concisas, suporte emocional e atendendo os seus desejos como mãe. Diante dos problemas supracitados, o presente estudo tem como objetivo abordar a respeito das práticas clínicas consideradas como violência obstétrica e ressaltar suas consequências na vida da mulher.

\section{REVISÃO BIBLIOGRÁFICA}

O parto é um marco importante na vida de uma mulher. Trata-se de um evento que necessita de cuidado e atenção, sendo o momento no qual a autonomia da gestante deva ser respeitada (BRASIL, 1996). No entanto, nem sempre foi dessa forma, sabe-se que, milhares de mulheres passaram por situações desumanas, devido algumas mudanças ao longo do tempo, onde a mulher deixou de estar no centro para os médicos assumirem os procedimentos (PALHARINI LA e FIGUEIRÔA SDM, 2018; VENDRUSCOLO CT, 2018).

Até o final do século XVIII, os partos eram considerados um ritual da mulher, realizado na privacidade dos domicílios da família com o acompanhamento de parteiras. No início do século XIX, inicia-se um processo de mudança, deixando de ser da esfera feminina e passando a ser de esfera médica, com tecnologias cada vez mais aplicadas (CAVALCANTE ACM, 2018). Esse episódio foi marcado por forte influência médica, uma vez que incluíam esse evento, que antes era fisiológico e natural, por um de risco e patológico como regra, e não mais com exceção (ZANARDO GLP, et al., 2017).

A implementação de técnicas na hora do parto de forma rotineira, como medicações, cesarianas, episiotomias, fórceps, manobra de Kristeller eram vistas pelo profissional de saúde como essencial para garantir uma "melhor assistência e um parto seguro" (VENDRUSCOLO CT, 2018). Porém, tem ocasionado uma grande insatisfação por parte das mulheres, por serem vítimas de uma opressão, principalmente quando surge o movimento social no final dos anos 80 , pela conquista de humanização do parto, com 0 intuito de melhorar esse modelo de médico no centro (DIAS MAB, et al., 2011; BRASIL, 2002).

Em 1985, a OMS propôs ações que incentivavam o parto via vaginal, livre movimentação e posição confortável, amamentação com o contato pele a pele logo após o nascimento e o direito de acompanhante durante todo o período de parto e pós-parto, para que fortalecesse o vínculo afetivo. Essa proposta teve como objetivo reduzir situações que prejudicavam à gestante e o bebê, como a ansiedade e o medo e, a partir daí, o parto passaria a ter ações humanizadas, com práticas menos agressivas, mais naturais e benéficas à fisiologia feminina, resgatando o protagonismo da mulher (BRASIL, 2002).

Nesse período, a expressão "violência obstétrica" começa a ser descoberto e representado por uma parte da sociedade, como na obra "Espelho de Vênus" de Edward Burne-Jones, que retratava um parto institucionalizado e impetuoso. Porém, o assunto foi ignorado por grande parcela da população, principalmente pelos profissionais de saúde em reconhecer a intervenção prestada como uma violência (DINIZ SG, et al., 2015). Assim, começou a crescer o conceito da violência contra mulheres nas instituições de saúde e com maior atenção sobre quatro tipos: negligência, violência psicológica, física e sexual (OLIVEIRA VJ e PENNA CMM, 2017). 
Além disso, a intensificação de intervenções desnecessárias no parto, acaba por emergir o conceito de violência obstétrica no campo da saúde, entendendo como qualquer ato dirigida a parturiente ou neonato, praticada sem o consentimento da mulher e/ou em desrespeito a sua autonomia, integridade física ou psicológica, indo contra os seus sentimentos e desejos (QUEIROZ FS, et al., 2020).

Na pesquisa "Nascer no Brasil" (LEAL MC e GAMA SGN, 2014) realizada no período de fevereiro/2011 a outubro/2012 com a aplicação do questionário em 23.940 mulheres no pós-parto, verificou-se o excesso de práticas no parto e nascimento, mostrando a incidência de intervenções prejudiciais que são realizadas nos hospitais sem necessidade, expondo as mulheres e seus recém-nascidos a efeitos adversos ou complicações resultantes das condutas médicas (LANSKY S, et al., 2014).

Os dados mostraram que 37\% das puérperas que participaram do estudo foram submetidas à manobra de Kristeller (técnica que consiste em pressionar a parte superior do útero para expulsar o bebê), procedimento agressivo e que pode causar lesões graves tanto na mãe quanto no bebê. A aplicação de ocitocina e ruptura artificial da membrana amniótica como medidas para acelerar o trabalho de parto foi realizado em $40 \%$ das gestantes. Cerca de mais da metade das mulheres relataram ser submetidas à episiotomia e $91,7 \%$ ficaram em posição de litotomia, mesmo quando é recomendado posições verticalizadas, como em pé e de cócoras (LANSKY S, et al., 2014; LEAL MC, et al., 2014).

A falta de informação e perda da autonomia tem sido alvo durante o trabalho de parto, pois diversos procedimentos são implementados sem a autorização da gestante ou muitas vezes, autorizadas, mas sem entender afundo aquela conduta, devido utilizarem termos científicos e complexos. Além disso, há casos em que há uma omissão da progressão de parto como a dilatação, vitalidade fetal e motivos irreais que deixam a mulher mais frágil, insegura e com medo de realizar um parto natural e humanizado, e acaba optando por cesariana, como os casos de circular de cordão umbilical (ROCHA NFF e FERREIRA J, 2020).

Acerca especificamente da violência verbal, são diversos fatores que influenciam para esse ato acontecer, como classe socioeconômica baixa, raça negra, orientação sexual, religião ou até mesmo o estado civil. Porém, a repercussão na parturiente é gigantesca, o sentimento de humilhação, perda de integridade e dignidade, desagradando o momento do parto e marcando-o negativamente. Alguns exemplos de frases violentas utilizadas por profissionais durante o trabalho de parto ironizam, desmoralizam e ameaçam a dor e a individualidade da mulher, ao supor que seu filho nasceria surdo por se expressar durante as contrações e o asco que seu marido sentiria ao vê-la naquela condição (SILVA MG, et al., 2014).

Tais exemplos convertem em parte do discurso institucional, e relaciona a dor do parto como punição que deve ser cumprida em prol do prazer da atividade, o que leva a uma trivialização do desrespeito a mulher e a invisibilidade da violência (MARTINS AC e BARROS GM, 2016). Portanto, existem uma série de ações não medicamentosas que podem ser utilizadas para reduzir a dor e o desconforto e ainda proporcionar uma experiência positiva no trabalho de parto, como os banhos de chuveiro ou de imersão, massagens na região lombar, respiração padronizada, condicionamento verbal e relaxamento (GALLO RBS, et al., 2011).

Segundo a OMS (1996), o parto é um evento natural e fisiológico, em que o corpo vai se preparando ao longo das semanas, para uma liberação adequada de hormônios que auxiliarão durante o parto, com uma boa dilatação e expulsão do bebê, e um melhor desfecho para a saúde materna e fetal (BRASIL, 1996). Porém, o número de cesarianas realizadas no Brasil só aumenta, como mostra no DATASUS nos anos de 2009-2019 com 55\% dos nascidos vivos, o que vai de desencontro a taxa ideal recomendadas pela a OMS (1985), que seria de 10 a $15 \%$ de todos os partos (FILHO MB e RISSIN A, 2018).

Sem dúvida as cesarianas representam um notável progresso na assistência obstétrica, porém, constantemente, estão sendo realizadas sem reais motivos, visto que, é economicamente mais interessante para médicos do setor privado, por economizarem tempo, trabalho e ganharem bem, já na rede pública essa prática auxilia contra, devido as superlotações (CUNHA CCC, 2015). Logo, as cesarianas devem ser realizadas apenas em casos que haja motivos médicos para a realização de tal procedimento, podendo muitas vezes salvar a vida da mãe e de seu filho (BRASIL, 2015). 
Complicações como aumento de morbimortalidade, internação em Unidade de Terapia Intensiva (UTI), uso de antibióticos, histerectomia, tempo de permanência no hospital, necessidade de transfusão em mulheres que fizeram parto cesáreo são maiores em relação aos partos vaginais (MASCARELLO KC, et al., 2017). Em gestações futuras, o parto cesariano pode aumentar o risco de placenta anômala e consequências para esses nascidos, como maior risco de mortalidade neonatal, prematuridade, uso de ventilação mecânica e comorbidade como diabetes tipo I, obesidade, asma, alergias de pele e digestiva, e outras relacionados ao metabolismo e sistema imunológico (TESSER CD, 2015).

Além disso, os procedimentos que são realizados durante o parto influenciam diretamente na saúde reprodutiva e sexual da mulher (BRASIL, 2002). Dados sobre partos de nulíparas apontam que lacerações do períneo foram mais frequentes em mulheres submetidas à episiotomia, levando ao pensamento de que 0 uso seletivo poderia minimizar traumas dos partos normais em nulíparas (COSTA NML, 2011).

Algumas das complicações envolvidas com a episiotomia abrangem o hematoma, rotura de períneo de $3^{\circ}$ e e $4^{\circ}$ graus, lesão do nervo pudendo, infeções, incontinência urinaria, dispareunia em longo prazo e piora na função sexual. (SOBIERAY NLEC e SOUZA BM, 2019). Dessa forma, deve-se avaliar os riscos e benefícios da episiotomia individualmente nas gestantes, evitando assim, o uso indiscriminado e prejudicial no parto (COSTA NML, 2011).

Dossiê da Violência Obstétrica (2012), aponta que a manobra de kristeller tem como consequência a aplicação elevada de vácuo extrator nos partos distócicos, além de causar na parturiente um desconforto e, não dispondo de uma certa comprovação cientifica que os benefícios superam os riscos materno-fetais, e realizem menos a episiotomia ou evitem casos de laceração de períneo (CARVALHO LCV, 2014).

Quanto à ocitocina, que é um medicamento para aumentar as frequências das contrações uterinas, e que está sendo utilizado de forma indiscriminada para acelerar o processo de parto, aumentando desnecessariamente as dores e as chances de ocorrer ruptura uterina (CUNHA CCC, 2015). Souza ASR, et al. (2010), aponta que a introdução de ocitocina pode levar a prolapso de cordão umbilical, falha da indução, prematuridade, infecção intracavitária, sofrimento e morte fetal. Ou seja, ela deve ser indicada quando realmente é necessária, uma vez que o corpo feminino é responsável por toda a preparação e mudança fisiológica para ter um parto vaginal (BRANDT GP, et al., 2018).

O parto instrumentalizado é aquele que utiliza fórceps ou vácuo extrator para auxiliar no período expulsivo em certas situações que são indicadas, não devendo ser feito de rotina (LEAL MC, et al., 2014). Hernández DH, et al. (2012), afirma que a utilização do fórceps tem sido relacionada a traumas obstétricos maternos (como aumento de lesões pélvicas, incontinência urinaria, sangramento retal e dor) e fetais (como fraturas ósseas, hemorragia intracanal e até lesões neurológicas). Pesquisas recentes indicam que grande parte dessas consequências se dá por falta de praticabilidade e inexperiência do obstetra, fazendo com que as gestantes tenham medo desse instrumento e por não levarem em conta as indicações propostas pelo Ministério da Saúde (CUNHA AA, 2011).

Assim, são muitos os fatores que levam ao desconforto das mulheres no processo de parto, entre eles o isolamento, uso de instrumentos, descaso dos profissionais em relação a queixa de dor, medo, desejos, expectativas, dentre outros (DIAS MAB, et al., 2011). Pode-se observar, que muitas mulheres que passaram por violência obstétrica possuem alguma sequela, seja relacionada a danos psicológicos ou físicos no momento do parto, refletindo em decisões futuras, a ter ou não ter mais filhos (BRANDT GP, et al., 2018).

O parto é o momento no qual a gestante necessita de mais apoio e compreensão, por esse motivo, é imprescindível o acompanhamento da família. A Lei do Acompanhante, lei n 11.108/05 aprovada no ano de 2005 no Brasil, garante à mulher a presença de um acompanhante durante o parto, considerando:

"Art. $1^{\circ}$ Regulamentar, em conformidade com o art. $1^{\circ}$ da Lei $n^{\circ} 11.108$, de 7 de abril de 2005, a presença de acompanhante para mulheres em trabalho de parto, parto e pós-parto imediato nos hospitais públicos e conveniados com o Sistema Único de Saúde - SUS" (BRASIL, 2005). 
De acordo com o Ministério da Saúde, o parto humanizado envolve desde o início da gestação até o nascimento, proporcionando autonomia feminina e planejamento reprodutivo. Sendo assim, é essencial que os profissionais perguntem sobre os desejos e expectativas, deixando a mulher à vontade e em um ambiente seguro, orientando-a sobre os possíveis procedimentos a serem realizados e inclui-la na tomada de decisões, sempre preservando o bem-estar físico e emocional (REZENDE CNV, 2014; ZASCIURINSKI MK, 2015). Assim, foram criados políticas e programas para que esse modelo de atenção obstétrica seja modificado, porém essa transformação ainda é um desafio que necessita de esforços de todos os envolvidos na saúde da mulher (BRASIL, 2011).

Políticas e programas estabelecidos pelo Ministério da Saúde nem sempre são colocadas em prática, retratando um crescimento do índice de violência obstétrica no Brasil (BRASIL, 2011). De acordo com a Fundação Perseu Abramo, 15\% das mulheres que realizaram o parto de forma natural afirmaram que sofreram desrespeito ou maltrato ao buscar assistência ou atendimento pré-natal. Quando se trata de diversas formas de violência obstétrica, $25 \%$ das mulheres relataram que sofreram entre uma a dez modalidades de violência durante o parto. Dentre essas violências, $10 \%$ refere ao exame de toque doloroso, $10 \%$ para negação do alívio de dor, $9 \%$ para não informação aos procedimentos adotados, $8 \%$ à negação de atendimento, e o restante são atos inaceitáveis dos profissionais, como gritos, xingamento e humilhações (TERRIBILI A e MANZANO M, 2011).

Portanto, as ações do Ministério da Saúde aliado a uma instrução de acadêmicos e profissionais de saúde quanto ao parto humanizado, são fundamentais para a erradicação de casos de violência obstétrica no Brasil (BRASIL, 2002). A diminuição dessa violação, se dá pelo conhecimento de práticas verdadeiramente humanizadas, pela utilização do plano de parto, por veiculação de informações sobre o período pré parto, intra parto e pós parto e pelo conhecimento sobre a utilização de manobras mais invasivas, somente se houver necessidade (GOOTTSCHALK, et al., 2018).

As intervenções pré-parto podem se tornar menos comuns com o auxílio do plano de parto, conversas e instrução da gestante e sua família, sendo realizadas em todas as consultas de pré-natal, programas e campanhas do Ministério da Saúde e profissional especializado em práticas humanizadas (BRASIL, 2002). Essas ações são capazes de diminuir dúvidas e anseios, diferenciar o verdadeiro e falso trabalho de parto, estimular e ressaltar a importância do parto vaginal e do acompanhamento, como também a realização de cesarianas e instrumentos complementares, se houver necessidade (MEDEIROS MSMF, et al., 2015).

Já o intraparto costuma ser o período em que mais acontecem violência, seja por excesso de práticas invasivas, forma de tratamento com a parturiente e/ou por falta de cuidados humanizados. As posições impostas, a falta de analgesia caso necessária e a falta de educação e empatia são ações que podem ser evitadas a partir de uma boa formação de profissionais humanizados e com a instrução da gestante no período que antecede o intra parto e no próprio período. (MEDEIROS MSMF, et al., 2015).

Em relação ao pós parto, as condutas humanizadas interferem diretamente na relação futura entre a mãe e o bebê, como o clampeamento tardio do cordão umbilical, o aleitamento materno e o contato pele a pele, garantindo a "hora dourada" ou "golden hour" (ANTUNES MB, et al., 2017). Essa "hora mágica" é o momento em que o bebê está mais atento e alerta, sendo importante incentivar o contato mãe-bebê, para que tenha uma boa amamentação na primeira hora de vida e, garantindo um vínculo entre o binômio. (BRANDT, et al., 2017).

Contudo, a violência obstétrica é o problema de saúde pública, uma vez que os números são acentuados e muitas vezes mascarados pela equipe profissional, necessitando assim, de intervenções políticas e culturais, principalmente, na formação de profissionais e gestores da saúde que sejam especializados em obstetrícia humanizada, tendo em vista a necessidade de mudança do sistema de atenção ao parto e nascimento (LANSKY S, et al., 2019).

Dessa forma, é necessário a participação ativa de profissionais instruídos sobre parto humanizado, juntamente a gestante amparada pela família e equipe médica, que busca uma qualidade na relação interpessoal médico-mãe-bebê de forma a criar um ambiente acolhedor. Além disso, é imprescindível uma 
mudança nos protocolos hospitalares por não incentivarem o parto natural, impor procedimentos e técnicas não benéficas e muitas vezes, utilizadas de forma desnecessárias, apenas para acelerar o trabalho de parto. Assim, é indispensável que ocorram mudanças no modelo obstétrico, em que equipe de saúde em conjunto com a família priorizem um plano de parto seguro, orgânico, multiprofissional e de acordo com os desejos da gestante, valorizando os aspectos emocionais, sociais e culturais desde a gestação até o puerpério, a fim de que as mulheres tenham a experiência de um parto mais humanizado.

\section{CONSIDERAÇÕES FINAIS}

Com o presente estudo, foi possível observar algumas práticas clínicas na hora do parto que são consideradas como violência obstétrica, porém, na maioria das vezes, mascaradas pelos profissionais de saúde. Logo, na obstetrícia é possível perceber um conjunto de violências (não necessariamente só físicas) direcionada a mulher no momento pré-parto, intra parto e pós-parto, subdividindo-se em alguns principais tipos de agressões, sendo elas: física, institucional, moral, sexual, psicológica e verbal, todas trazendo consigo sequelas na vida reprodutiva/sexual da mulher. Contudo, foi percebido que é primordial uma boa relação médico-paciente, a qual deve conter confiança e acima de tudo diálogo, sempre mantendo a gestante informada e com autonomia sobre seu corpo.

\section{REFERÊNCIAS}

1. ANTUNES MB, et al. Amamentação na primeira hora de vida: conhecimento e prática da equipe multiprofissional. Av Enfermagem, 2017; 35(1): 19-29.

2. BRANDT GP, et al. Violência obstétrica: a verdadeira dor do parto. Revista Gestão e Saúde. 2018; 19-37;

3. BRASIL. Lei № 11.108, de 7 de Abril de 2005. Altera a Lei no 8.080, de 19 de setembro de 1990, para garantir às parturientes o direito à presença de acompanhante durante o trabalho de parto, parto e pós-parto imediato, no âmbito do Sistema Único de Saúde - SUS. Diário Oficial da União, Brasília-DF, 2005.

4. CARVALHO LCV. Os efeitos da manobra de Kristeller no segundo período de trabalho de parto. Dissertação (Mestrado em Enfermagem de Saúde Materna e Obstetrícia) - Escola Superior de Enfermagem de Porto. Porto, 2014; 92 p.

5. CAVALCANTE ACM, et al. Violência obstétrica: desvelando suas consequências. Investigação Qualitativa em Saúde, 2018; 2.

6. COSTA NML, et al. Episiotomia nos partos normais: uma revisão de literatura. Revista de Ciências da Saúde Nova Esperança, 2011; 9(2): 46-51.

7. CUNHA AA. Indicações do parto a fórceps. Revista Femina, 2011; 39(12).

8. CUNHA CCC. Violência obstétrica: uma análise sob o prisma dos direitos fundamentais. Tese (UnB) - Brasília, 2015.

9. DIAS MAB, et al. Humanização do parto: política pública, comportamento organizacional e ethos profissional. Caderno de Saúde Pública, RJ, 2011; 27(5), 1042-1043;

10. DINIZ SG, et al. Violência obstétrica como questão de saúde pública no Brasil: origens, definições, tipologia, impactos sobre a saúde materna e propostas para sua prevenção. Journal of Human Growth and Develop, 2015; 25(3): 377-376.

11. FILHO MB, RISSIN A. A OMS e a epidemia de cesarianas. Revista Brasileira de Saúde materno Infantil, 2018; 18(1): 3-4.

12. GALLO RBS, et al. Recursos não-farmacológicos no trabalho de parto: protocolo assistencial. Revista Femina, 2011; $39(1): 42-48$.

13. HERNÁNDEZ DH, et al. Complicaciones maternas y neonatales secundarias a parto vaginal instrumentado con fórceps. Revista de Investigación Médica Sur, 2012; 19(2): 52-55.

14. LANSKY S, et al. Pesquisa nascer no Brasil: perfil da mortalidade neonatal e avaliação da assistência à gestante e ao recém-nascido. Caderno de Saúde Pública, 2014;

15. LANSKY S, et al. Violência obstétrica: influência da Exposição Sentidos do Nascer na vivência das gestantes. Ciência e Saúde Coletiva, 2019; 24 (8).

16. LEAL MC, et al. Intervenções obstétricas durante o trabalho de parto e parto em mulheres brasileiras de risco habitual. Cadernos de Saúde Pública, 2014; 30(Supl. 1):17-32.

17. LEAL MC, GAMA SGN. Nascer no Brasil. Caderno de Saúde Pública, 2014; 30(1): S5.

18. MARTINS AC, BARROS GM. Parirás na dor? Revisão integrativa da violência obstétrica em unidades públicas brasileiras. Revista Dor, 2016; 17(3): 215-218.

19. MASCARELLO KC, et al. Complicações maternas e cesárea sem indicação: revisão sistemática e meta-análise. Revista de Saúde Pública, 2017; 51: 105. 
20. MEDEIROS MSMF, et al. Humanização do Trabalho de parto e nascimento: aplicações de estratégias não farmacológicas efetivas nesse processo. Revista de Enfermagem, 2015; 9(7): 9133-9138.

21. ORGANIZAÇÃO MUNDIAL DE SAÚDE (OMS). Declaração da OMS sobre Taxas de Cesáreas. 2015. Disponível em: http://apps.who.int/iris/bitstream/handle/10665/161442/WHO_RHR_15.02_por.pdf;jsessionid=7D47289ED6626FEA1 CC6823C64767932?sequence=3. Acessado em: 20 mar 2021.

22. ORGANIZAÇÃO MUNDIAL DE SAÚDE (OMS). Maternidade segura: assistência ao parto normal: um guia prático. 1996. Disponível em: https://saude.mppr.mp.br/arquivos/File/kit_atencao_perinatal/manuais/assistencia_ao_parto_normal_2009.pdf. Acessado em: 27 ago 2021.

23. ORGANIZAÇÃO MUNDIAL DE SAÚDE (OMS). Parto e nascimento domiciliar assistido por parteiras tradicionais. 2010. Disponível em: https://bvsms.saude.gov.br/bvs/publicacoes/parto_nascimento_domiciliar_parteiras.pdf. Acessado em: 20 mar 2021.

24. ORGANIZAÇÃO MUNDIAL DE SAÚDE (OMS). Portaria №. 1.459, de 24 de Junho de 2011. 2011. Disponível em: https://bvsms.saude.gov.br/bvs/saudelegis/gm/2011/prt1459_24_06_2011.html. Acessado em: 20 mar 2021.

25. ORGANIZAÇÃO MUNDIAL DE SAÚDE (OMS). Programa Humanização do Parto: humanização no pré-natal e nascimento. 2002. Disponível em: https://bvsms.saude.gov.br/bvs/publicacoes/parto.pdf. Acessado em: 20 mar 2021.

26. OLIVEIRA VJ, PENNA CMM. O discurso da violência obstétrica na voz das mulheres e dos profissionais de saúde. Tese - enfermagem, Florianópolis, 2017; 26(2).

27. PALHARINI LA, FIGUEIRÔA SDM. Gênero, história e medicalização do parto: a exposição "Mulheres e práticas de saúde". História, Ciências e Saúde, 2018; 25(4): 1039-1061.

28. QUEIROZ FS, et al. Obstetric violence: a public healt problem and a violation of human rights. Journal of health review, 2020; 3(5): 14435-14445.

29. REZENDE CNV. Violência obstétrica: uma ofensa a direitos humanos ainda não reconhecida legalmente no brasil. Dissertação - UniCEUB, Brasília, 2014;

30. ROCHA NFF, FERREIRA J. A escolha da via de parto e a autonomia das mulheres no Brasil: uma revisão integrativa. Revista Saúde Debate, 2020; 44(125): 556-568;

31. SILVA MG, et al. Violência Obstétrica na visão das Enfermeiras Obstetras. Revista Rene, 2014; 15(4): 820-8.

32. SOBIERAY NLEC, SOUZA BM. Prevalência de episiotomia e complicações perineais quando da sua realização ou não em uma maternidade de baixo risco do complexo HC/UFPR. Arq Med Hosp Fac Cienc Med Santa Casa São Paulo, 2019; 64(2): 93-99.

33. SOUZA ASR, et al. Indução do trabalho de parto: conceitos e particularidades. Revista Femina, 2010; 38(4).

34. TERRIBILI A, MANZANO M. Gravidez, filhos e violência institucional no parto. Fundação Perseu Abramo, 2011.

35. TESSER CD et al. Violência obstétrica e prevenção quaternária: o que é e o que fazer. Revista Brasileira de Medicina da Família e da Comunidade, 2015; 10(35);

36. VENDRUSCOLO CT. A história do parto: do domicilio ao hospital das parteiras; das parteiras ao médico; de sujeito a objeto. Dissertação (psicologia) - Centro Universitário Franciscano. Ciências Humanas, 2016; 16(1): 95-107.

37. VENTURI G, GODINHO T. Mulheres brasileiras e gênero nos espaços público e privado: uma década de mudanças na opinião pública. São Paulo: Editora Fundação Perseu Abramo, SESC-SP, 2013;

38. ZANARDO GLP, et al. Violência Obstétrica No Brasil: Uma Revisão Narrativa. Psicologia e Sociedade, 2017; 29: 111.

39. ZASCIURINSKI MJ. Violência obstétrica: uma contribuição para o debate acerca do empoderamento feminino. Tese, 2015. 